\title{
Photoionization of Ca XV with high energy features
}

\author{
Sultana N. Nahar \\ Department of Astronomy, The Ohio State University, Columbus, OH 43210, USA \\ Email: nahar.1@osu.edu, tel: 1-614-292-1888,fax: 1-614-292-2928
}

\begin{abstract}
Photoionization cross sections of $(\mathrm{Ca} \mathrm{XV}+h \nu \rightarrow \mathrm{Ca} \mathrm{XVI}+\mathrm{e})$, with high energy resonant photo-absorption phenomena, of a large number of bound states, 701 in total with $n \leq 10$ and $l \leq 9$, are reported. They are obtained using the R-matrix method with a close coupling (CC) wavefunction expansion of 29 states of $n=2,3$ complexes of the core ion Ca XVI. Characteristic features found in photoionization of the ion are illustrated with examples. The cross section $\left(\sigma_{P I}\right)$ of the ground $2 s^{2} 2 p^{2}\left({ }^{3} P\right)$ state is found to be unaffected by the size of the wavefunction expansion except for weak sparse resonances in high energy region. However, effects on excited states are considerable as the core excitations to $\mathrm{n}=3$ states are manifested in huge resonant absorption in high energy photoionization. They show existence of prominent high peak resonant features and enhancement in the background that were not studied before for Ca XV. In addition photoionization of the excited states with a single valence electron is dominated by Seaton resonant structures formed by the photo-excitation-ofcore in the high energy region. These features will impact other quantities, such as the opacity, electron-ion recombination in high temperature plasmas where the ion exists, and hence will play important role in determination of elemental abundances in the astronomical objects.
\end{abstract}

Keywords: Photoionization of Ca XV, cross sections of excited levels, R-matrix method, resonant features 32.10-f,95.30.Ky,32.80-t

\section{Introduction}

Ca XV, a highly charged carbon-like ion that typically exists in high temperature plasmas (e.g. Emission Line Coronal Photometer (ELCP) at http://nsosp.nso.edu/corona) plays an important role for diagnostics and modeling. For example, the diagnostic emission lines of $\mathrm{Ca} \mathrm{XV}$ in the solar extreme ultraviolet spectra were

Email addresses: nahar.1@osu.edu (Sultana N. Nahar), Tel:1-614-292-1888, Fax: 1-292-2928 (Sultana N. Nahar)

URL: http://www.astronomy.ohio-state.edu/ nahar (Sultana N. Nahar)

Preprint submitted to New Astronomy

August 12, 2016

(C) 2016. This manuscript version is made available under the Elsevier user license http://www.elsevier.com/open-access/userlicense/1.0/ 
studied by Dere [1], Laming and Drake [2], were detected in star $(\Xi)$ Bootis spectrum by the Extreme- Ultraviolet Explorer satellite [2], were studied by Keenan et al. [3], Aggarwal and Keenan [4]. Photoionization of Ca XV was studied earlier by Luo and Pradhan [5] and Nahar and Pradhan [6] under the Opacity Project $[7,8]$. Both of these studies used R-matrix method, but using a smaller wavefunction expansion of 8-CC, that is, included $8 \mathrm{LS}$ states from $\mathrm{n}=2$ complex of the core ion Ca XVI. It has been found that core excitations to high lying states can be more dominant in high energy photoionization than those with the low lying states (e.g. for Fe XVII $[9,10]$ ). Hence it is important to study the possible high core excitations and impact in the high energy region for this highly charged ion. The present works reports such study using a 29-CC wavefunction expansion that includes excitations to both $\mathrm{n}=2$ and 3 complexes.

\section{Theory and Computations}

The details of the process of photoionization and R-matrix method with close coupling approximation for the wavefunction are available in literature (e.g. [11]). Hence only a brief theoretical outline is presented below. Photoionization of an atomic system can be direct as

$$
h \nu+X^{+} \rightarrow e+X^{++},
$$

and through a two-step process where the intermediate state is a doubly excited autoionizing state

$$
X^{+}+h \nu \leftrightarrow\left(X^{+}\right)^{* *} \leftrightarrow X^{++}+e
$$

The autoionizing state leads either to autoionization where the electron goes free or to dielectronic recombination where the electron is captured by emission of a photon (e.g. [11]). This two-step process introduces a resonance in photoionization.

Theoretically the doubly excited autoionizing state can be described by including the core excitation in the wavefunction, as considered in the close coupling (CC) approximation. In this approximation the atomic system is described by a 'target' or 'core' ion of N-electrons interacting with the $(\mathrm{N}+1)$ th electron. The $(\mathrm{N}+1)$ th electron is bound if the energy $\mathrm{E}$ is negative or in the continuum if energy is positive. The total wavefunction, $\Psi_{E}$, of the $(\mathrm{N}+1)$ electrons system in a symmetry $S L \pi$ is represented by the core eigenfunction, $\chi_{i}$, coupled with the $(\mathrm{N}+1)$ th electron function, $\theta_{i}$. as

$$
\Psi_{E}(e+i o n)=A \sum_{i} \chi_{i}(\text { ion }) \theta_{i}+\sum_{j} c_{j} \Phi_{j}
$$

The sum in the first term is over the number of core states. The $(N+1)$ th electron with kinetic energy $k_{i}^{2}$ is in a channel labeled as $S_{i} L_{i} \pi_{i} k_{i}^{2} \ell_{i}(S L \pi)$ where the core state is $S_{i} L_{i} \pi_{i}$. The second sum, basically part of the first sum, accounts for short range correlation and the orthogonality between the continuum and the 
bound electron orbitals. $\Phi_{j}$ s are bound channel functions of the $(\mathrm{N}+1)$-electrons system. Substitution of $\Psi_{E}(e+i o n)$ in the Schrodinger equation

$$
H_{N+1} \Psi_{E}=E \Psi_{E}
$$

introduces a set of coupled equations that are solved using the R-matrix approach (e.g. $[12,11]$. The solution is a continuum wavefunction, $\Psi_{F}$, for an electron with positive energy $(\mathrm{E}>0)$, or a bound state, $\Psi_{B}$, at a negative total energy $(\mathrm{E} \leq 0)$. The present calculations are carried out in LS coupling approximation where the Hamiltonian of the $(\mathrm{N}+1)$-electrons system is given by

$$
H_{N+1}=\sum_{i=1}^{N+1}\left\{-\nabla_{i}^{2}-\frac{2 Z}{r_{i}}+\sum_{j>i}^{N+1} \frac{2}{r_{i j}}\right\},
$$

Calculations included the relativistic mass correction and Darwin terms as adopted under the Iron Project [13].

The transition matrix elements for photoionization, $\left\langle\Psi_{B}\|\mathbf{D}\| \Psi_{F}\right\rangle$ where $\mathbf{D}=\sum_{i} r_{i}$ is the dipole operator and the sum is over the number of electrons, are obtained from the bound and continuum wavefunctions. The transition matrix element is reduced to generalized line strength as

$$
\mathbf{S}=\left|\left\langle\Psi_{f}\|\mathbf{D}\| \Psi_{i}\right\rangle\right|^{2}=\left|\left\langle\psi_{f}\left|\sum_{j=1}^{N+1} r_{j}\right| \psi_{i}\right\rangle\right|^{2},
$$

where $\Psi_{i}$ and $\Psi_{f}$ are the initial and final state wavefunctions. The photoionization cross section $\left(\sigma_{P I}\right)$ is proportional to the generalized line strength as

$$
\sigma_{P I}=\frac{4 \pi^{2}}{3 c} \frac{1}{g_{i}} \omega \mathbf{S},
$$

where $g_{i}$ is the statistical weight factor of the bound state and $\omega$ is the incident photon energy. The complex resonant structures in photoionization result from couplings between the continuum channels and bound channels in transition matrix. They appear at electron energies $k_{i}^{2}$ corresponding to autoionizing states of the Rydberg series, $S_{i} L_{i} \pi_{i} \nu \ell$ where $\nu$ is the effective quantum number, belonging to excited target or core state $S_{i} L_{i} \pi_{i}$.

\section{Computation}

Photoionization cross sections of Ca XV were computed using the R-matrix codes $[14,15]$ adopted and developed under the Opacity Project and the Iron Project $[8,13]$. The calculated energies were identified with spectroscopic designation using quantum defect analysis and percentage of channel contributions to the states using code ELEVID [18]. The cross section files were processed with code PRCLS [18]. 
Table 1: LS states and energies of the 29 LS states $\left(E_{c}\right)$ of the core ion Ca XVI included in the wavefunction expansion of Ca XV. The energies are compared with those at NIST compiled table [20]. The states were optimized using a set of 14 spectroscopic configurations: $2 s^{2} 2 p(1)$, $2 s 2 p^{2}(2), 2 p^{3}(3), 2 s^{2} 3 s(4), 2 s^{2} 3 p(5), 2 s^{2} 3 d(6), 2 s 2 p 3 s(7), 2 s 2 p 3 p(8), 2 s 2 p 3 d(9), 2 p^{2} 3 s(10)$, $2 p^{2} 3 p(11), 2 p^{2} 3 d(12), 2 s 3 p^{2}(13), 2 s 3 d^{2}(14)$, with filled $1 s^{2}$ orbital. The Thomas-Fermi scaling parameters for the orbitals are $2.40(1 \mathrm{~s}), 1.37(2 \mathrm{~s}), 1.45(2 \mathrm{p}), 1.40(3 \mathrm{~s}), 1.15(3 \mathrm{p}), 1.10(3 \mathrm{~d})$.

\begin{tabular}{|c|c|c|c|c|}
\hline & \multicolumn{2}{|c|}{ State } & $E_{c}(\mathrm{Ry})$ & $E_{o}(\mathrm{Ry})$ \\
\hline 1 & $2 s^{2} 2 p$ & ${ }^{2} P^{o}$ & 0.0 & 0. \\
\hline 2 & $2 s 2 p^{2}$ & ${ }^{4} P$ & 2.1611 & 2.635673 \\
\hline 3 & $2 s 2 p^{2}$ & ${ }^{2} D$ & 3.9755 & 4.382138 \\
\hline 4 & $2 s 2 p^{2}$ & ${ }^{2} S$ & 5.0983 & 5.396342 \\
\hline 5 & $2 s 2 p^{2}$ & ${ }^{2} P$ & 5.4197 & 5.847541 \\
\hline 6 & $2 p^{3}$ & ${ }^{4} S^{\circ}$ & 6.9544 & 7.607805 \\
\hline 7 & $2 p^{3}$ & ${ }^{2} D^{\circ}$ & 8.0242 & 8.591609 \\
\hline 8 & $2 p^{3}$ & ${ }^{2} P^{o}$ & 9.0417 & 9.649590 \\
\hline 9 & $2 s^{2} 3 s$ & ${ }^{2} S$ & 39.8154 & \\
\hline 10 & $2 s^{2} 3 p$ & ${ }^{2} P^{o}$ & 40.9909 & \\
\hline 11 & $2 s 2 p 3 s$ & ${ }^{4} P^{o}$ & 42.1537 & \\
\hline 12 & $2 s^{2} 3 d$ & ${ }^{2} D$ & 42.1119 & 42.49421 \\
\hline 13 & $2 s 2 p 3 s$ & ${ }^{2} P^{o}$ & 42.7540 & \\
\hline 14 & $2 s 2 p 3 p$ & ${ }^{4} D$ & 43.2337 & \\
\hline 15 & $2 s 2 p 3 p$ & ${ }^{4} S$ & 43.4265 & \\
\hline 16 & $2 s 2 p 3 p$ & ${ }^{4} \mathrm{P}$ & 43.6088 & \\
\hline 17 & $2 s 2 p 3 p$ & ${ }^{2} P$ & 43.1878 & 43.62236 \\
\hline 18 & $2 s 2 p 3 p$ & ${ }^{2} D$ & 43.7929 & \\
\hline 19 & $2 s 2 p 3 p$ & ${ }^{2} S$ & 44.1497 & \\
\hline 20 & $2 s 2 p 3 d$ & ${ }^{4} F^{o}$ & 44.1611 & \\
\hline 21 & $2 s 2 p 3 d$ & ${ }^{2} D^{\circ}$ & 44.4578 & \\
\hline 22 & $2 s 2 p 3 s$ & ${ }^{2} P^{o}$ & 44.4652 & \\
\hline 23 & $2 s 2 p 3 d$ & ${ }^{4} D^{\circ}$ & 44.4125 & 45.02672 \\
\hline 24 & $2 s 2 p 3 d$ & ${ }^{2} P^{o}$ & 45.1593 & \\
\hline 25 & $2 s 2 p 3 d$ & ${ }^{4} P^{o}$ & 44.5108 & 45.2262 \\
\hline 26 & $2 s 2 p 3 p$ & ${ }^{2} P$ & 45.5712 & \\
\hline 27 & $2 s 2 p 3 p$ & ${ }^{2} D$ & 45.6114 & \\
\hline 28 & $2 s 2 p 3 d$ & ${ }^{2} F^{o}$ & 45.0666 & 45.67792 \\
\hline 29 & $2 s 2 p 3 p$ & ${ }^{2} S$ & 45.9174 & \\
\hline
\end{tabular}

The Ca XVI core wavefunction was obtained from atomic structure calculations using the later version [16] of atomic structure code SUPERSTRUCTURE (SS) [17] which implements Thomas-Fermi-Dirac-Amaldi approximation. A set of 14 configurations of Ca XVI, listed in Table 1, was optimized for the orbital wavefunctions. The Thomas-Fermi scaling parameters for the orbitals are also given in the table. The calculated energies are compared with those from Sugar and Corliss [19] listed at NIST website [20]. These calculated Ca XVI energies are about the same as those used for 8-CC calculations by Nahar and Pradhan [6]. The observed energies of fine structure level energies were statistically averaged to obtain the LS term energies. The comparison shown in Table 1 is good in general except for a few states where the difference is large. It may be noted that results from a R-matrix calculation are often less sensitive to core energies because of achieving higher accuracy from interaction of a large number of configurations for the ion.

The wavefunction expansion included partial waves of the interacting elec- 
tron with orbital angular momenta of $0 \leq \ell \leq 9$. The R-matrix basis set of each orbital included 14 continuum functions within a boundary of 3 a.u.. The second term of the wavefunction in Eq. (3), which represents the bound state correlation functions, included all possible configurations with orbital occupancies from minimum to a maximum number as given within parentheses of the orbitals $2 \mathrm{~s}(0-2), 2 \mathrm{p}(0-4), 3 \mathrm{~s}(0-2), 3 \mathrm{p}(0-2), 3 \mathrm{~d}(0-2)$ with filled core shell $1 s^{2}$ for each configuration. Computations are carried out for all angular momenta, 0 $\leq L \leq 11$ of quintets, triplets and singlets with even and odd parity.

\section{Results and Discussions}

Photoionization cross sections of $\mathrm{Ca} \mathrm{XV}$ is reported for all bound states with $\mathrm{n} \leq 10$ obtained from R-matrix method. They include more complete features encompassing a high energy region of core excitations to $\mathrm{n}=3$ complex going up to 46 Ry compared to earlier study with excitations in $n=2$ complex up to energy 9.7 Ry. Features from $n=3$ complex is found to be much more significant than those from $n=2$ complex. They are discussed with illustrations below.

The number of bound states, 701 in total, obtained from 29-CC calculations is the same as that from 8-CC calculations [6]. The reason is that no bound state can be formed with core excitations beyond $\mathrm{n}=2$ states. These higher excitations lie above the ionization threshold. Table 2 presents comparison of the bound states from 29-CC calculations with those from 8-CC, and with limited number of energies available from Sugar and Corliss [19] listed in NIST compilation table. The comparisons show that energies from 8-CC and 29CC agree with each other very well indicating inclusion of higher channels had insignificant effect on the bound states. Energies from both 8-CC and 29-CC calculations agree very well, about or less than $1.5 \%$, with those at NIST [20].

As explained above that the resonances are formed by core excitations. For the highly charged ion carbon-like ion $\mathrm{Ca} \mathrm{XV}$ which exists in high temperature plasmas, it is very likely that the core would be excited to high lying states. This work focuses on the characteristic features formed from excitations of the core to these highly lying states. The study finds that these excitations have little effect on the $2 s^{2} 2 p^{2}\left({ }^{3} P\right)$ ground state photoionization of Ca XV. Figure 1 displays photoionization cross sections $\sigma_{P I}$ of the state over a large energy range obtained from the present 29-CC (panel a) and earlier 8-CC calculations (panel b) [6]. Resonances in $\sigma_{P I}$ and the background in both panels look very similar. The arrows in the panels point photoionization with limits for the core excitation to 8 states of $n=2$ complex and to 29 states going up to $3 d$ of $n=$ 3 complex. Some differences in resonance heights below 8-CC energy limit are due to energy resolution used in the computations. Figure shows sparse weak resonances and slight rise in the background for the 29-CC calculations.

In contrast to the ground state, the core excitations to high lying states have considerable impact on photoionization of excited states of Ca XV. They introduce (i) prominent resonances with high peaks and (ii) enhancement in the background in the high energy region. Examples of the effects are shown in two relatively low lying excited states, (a) $2 s^{2} 2 p^{2} P^{o} 3 s\left({ }^{3} P^{o}\right)$ and (b) $2 s 2 p^{2}{ }^{2} S 3 s\left({ }^{1} S\right)$, 


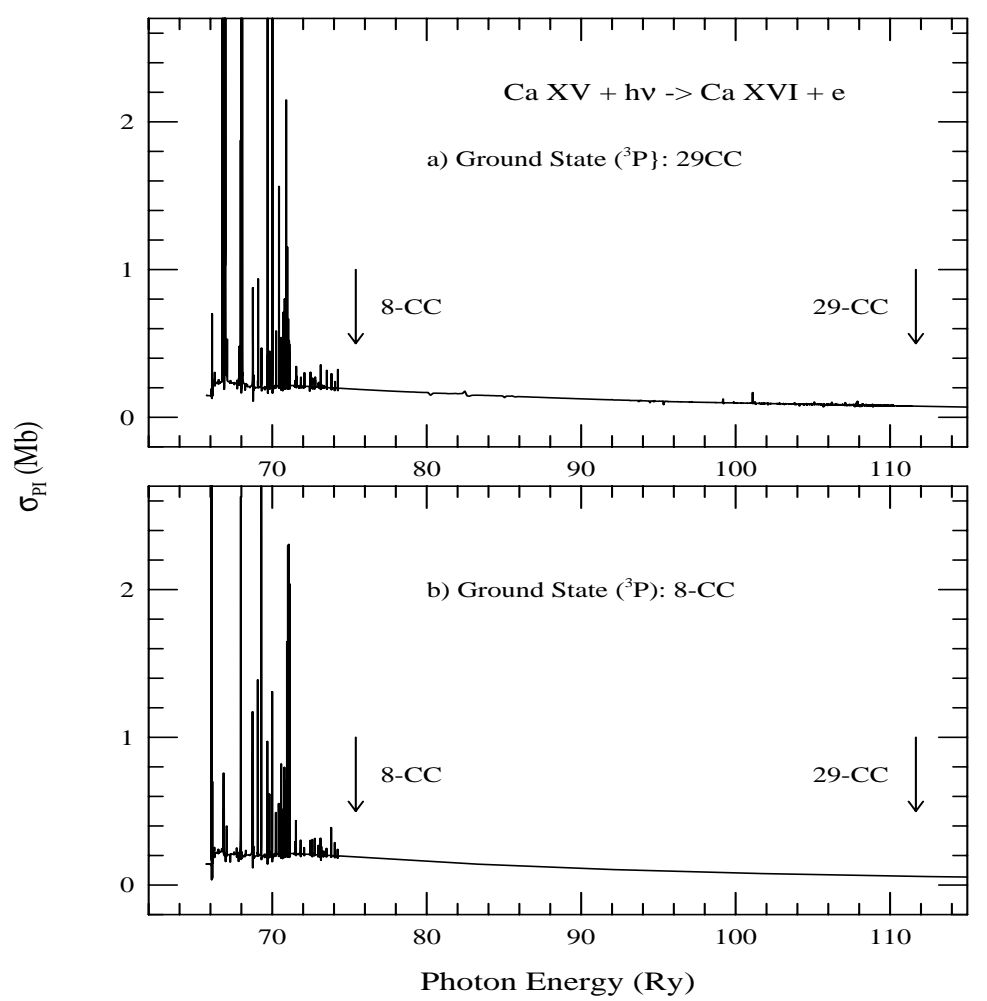

Figure 1: Comparison of photoionization cross sections $\sigma_{P I}$ of the ground $2 s^{2} 2 p^{2}\left({ }^{3} P\right)$ state of $\mathrm{Ca}$ XV obtained from (a) 29-CC and (b) 8-CC [6] calculations. The arrows point the photoionization energy limits with core excitations to $n=2$ and 3 complexes. The effect of excitations to $\mathrm{n}=3$ states in 29 - $\mathrm{CC}$ is insignificant 
Table 2: Comparison of calculated LS term energies, $E_{c}$, of Ca XV from 29-CC calculations with those from 8-CC and those $E_{o}$ from NIST [20]. NIST term energies were obtained from statistical averaging of fine structure energies at NIST [20]. All energies agree very well with each other.

\begin{tabular}{llccc}
\hline \multicolumn{1}{c}{ Conf } & Term & $E_{o}(\mathrm{Ry})$ & \multicolumn{2}{c}{$E_{c}(\mathrm{Ry})$} \\
& & & $8-\mathrm{CC}$ & $29 \mathrm{CC}$ \\
\hline $2 s^{2} 2 p^{2}$ & ${ }^{3} P$ & 65.512727 & $6.574 \mathrm{E}+01$ & $6.576 \mathrm{E}+01$ \\
$2 s^{2} 2 p^{2}$ & ${ }^{1} D$ & 64.758291 & $6.503 \mathrm{E}+01$ & $6.504 \mathrm{E}+01$ \\
$2 s^{2} 2 p^{2}$ & ${ }^{1} S$ & 63.946625 & $6.423 \mathrm{E}+01$ & $6.423 \mathrm{E}+01$ \\
$2 s\left({ }^{2} S\right) \cdot 2 p^{3} \cdot\left({ }^{4} S^{o}\right)$ & ${ }^{5} S^{o}$ & 63.233741 & $6.348 \mathrm{E}+01$ & $6.348 \mathrm{E}+01$ \\
$2 s\left({ }^{2} S\right) \cdot 2 p^{3} \cdot\left(\cdot{ }^{2} D^{o}\right)$ & ${ }^{3} D^{o}$ & 61.205126 & $6.140 \mathrm{E}+01$ & $6.140 \mathrm{E}+01$ \\
$2 s\left({ }^{2} S\right) \cdot 2 p^{3} \cdot\left(\cdot{ }^{2} P^{o}\right)$ & ${ }^{3} P^{o}$ & 60.423676 & $6.066 \mathrm{E}+01$ & $6.066 \mathrm{E}+01$ \\
$2 s\left({ }^{2} S\right) .2 p^{3} \cdot\left({ }^{4} S^{o}\right)$ & ${ }^{3} S^{o}$ & 59.105883 & $5.925 \mathrm{E}+01$ & $5.924 \mathrm{E}+01$ \\
$2 s\left({ }^{2} S\right) .2 p^{3} \cdot\left({ }^{2} D^{o}\right)$ & ${ }^{1} D^{o}$ & 59.098866 & $5.929 \mathrm{E}+01$ & $5.929 \mathrm{E}+01$ \\
$2 s\left({ }^{2} S\right) .2 p^{3} \cdot\left({ }^{2} P^{o}\right)$ & ${ }^{1} P^{o}$ & 58.326749 & $5.854 \mathrm{E}+01$ & $5.853 \mathrm{E}+01$ \\
$2 p^{4}$ & ${ }^{3} P$ & 55.542474 & $5.570 \mathrm{E}+01$ & $5.564 \mathrm{E}+01$ \\
$2 p^{4}$ & ${ }^{1} D$ & 54.857191 & $5.505 \mathrm{E}+01$ & $5.499 \mathrm{E}+01$ \\
$2 p^{4}$ & ${ }^{1} S$ & 53.437710 & $5.363 \mathrm{E}+01$ & $5.353 \mathrm{E}+01$ \\
$2 s^{2} 2 p .3 d$ & ${ }^{3} F^{o} *$ & 25.901023 & $2.599 \mathrm{E}+01$ & $2.600 \mathrm{E}+01$ \\
$2 s^{2} 2 p .3 d$ & ${ }^{3} D^{o}$ & 25.505882 & $2.571 \mathrm{E}+01$ & $2.572 \mathrm{E}+01$ \\
$2 s^{2} 2 p .3 d$ & ${ }^{3} P^{o} *$ & 25.332658 & $2.564 \mathrm{E}+01$ & $2.566 \mathrm{E}+01$ \\
$2 s^{2} 2 p .3 d$ & ${ }^{1} P^{o}$ & 24.983301 & $2.523 \mathrm{E}+01$ & $2.525 \mathrm{E}+01$ \\
$2 s^{2} 2 p .3 d$ & ${ }^{1} F^{o}$ & 24.968721 & $2.523 \mathrm{E}+01$ & $2.524 \mathrm{E}+01$ \\
$2 s 2 p^{2}\left({ }^{4} P\right) .3 d$ & ${ }^{3} F *$ & 22.674150 & $2.286 \mathrm{E}+01$ & $2.301 \mathrm{E}+01$ \\
\hline
\end{tabular}

in Figure 2. Similar to Figure 1, the panels have arrows marking the energy limits for $\mathrm{n}=2$ excitations for 8 - $\mathrm{CC}$ and $\mathrm{n}=3$ excitations for 29-CC calculations. It is obvious that the resonant structures between limits of $n=2$ and 3 complexes arsing from $\mathrm{n}=3$ states for both states are much more dominating that those below $n=2$ limits arising from states of $n=2$ complex. There are larger number of strong resonances with high peaks indicating much more photo-absorption in the high energy region than in the low energy region below $\mathrm{n}=2$ excitations. The importance of the impact can be realized in $\sigma_{P I}$ of the excited state $2 s 2 p^{2}{ }^{2} S 3 s\left({ }^{1} S\right.$ ) (b) of $\mathrm{Ca} \mathrm{XV}$ which has resonances belonging to $\mathrm{n}=2$ core states in the low energy region, but on the average the background is very low. Hence without inclusion of the high energy enhancements from $\mathrm{n}=3$ excitations, the state will contribute poorly for high temperature plasmas. The background enhancement adds to the resonant photo-absorption and without it the cross section would be underestimated by smooth decay with energy. These enhancements typically relate to photoionization leaving the residual ion in an excited state that is allowed for the core ground state for a dipole transition. The background enhancement for the excited state $2 s^{2} 2 p^{2} P^{o} 3 s\left({ }^{3} P^{o}\right)$ (a) of $\mathrm{Ca} \mathrm{XV}$ can be seen around energies $66.7 \mathrm{Ry}, 70 \mathrm{Ry}$ and $73 \mathrm{Ry}$. These are approximately $38 \mathrm{Ry}$, over $41 \mathrm{Ry}$, and $44 \mathrm{Ry}$ from the ionization threshold and correspond to states, such as ${ }^{2} S,{ }^{2} D,{ }^{2} P$ states (Table 1 ). These state are allowed for dipole transitions by the $2 s^{2} p\left({ }^{2} P^{o}\right)$ core ground state. The energy positions are not at the exact excitation energies as typically seen in few electron systems, such as, Fe XXIV [21]. A possible reason is the effect of electron- electron correlation on the interacting channels.

The other distinct resonant feature typically seen in photoionization of ex- 


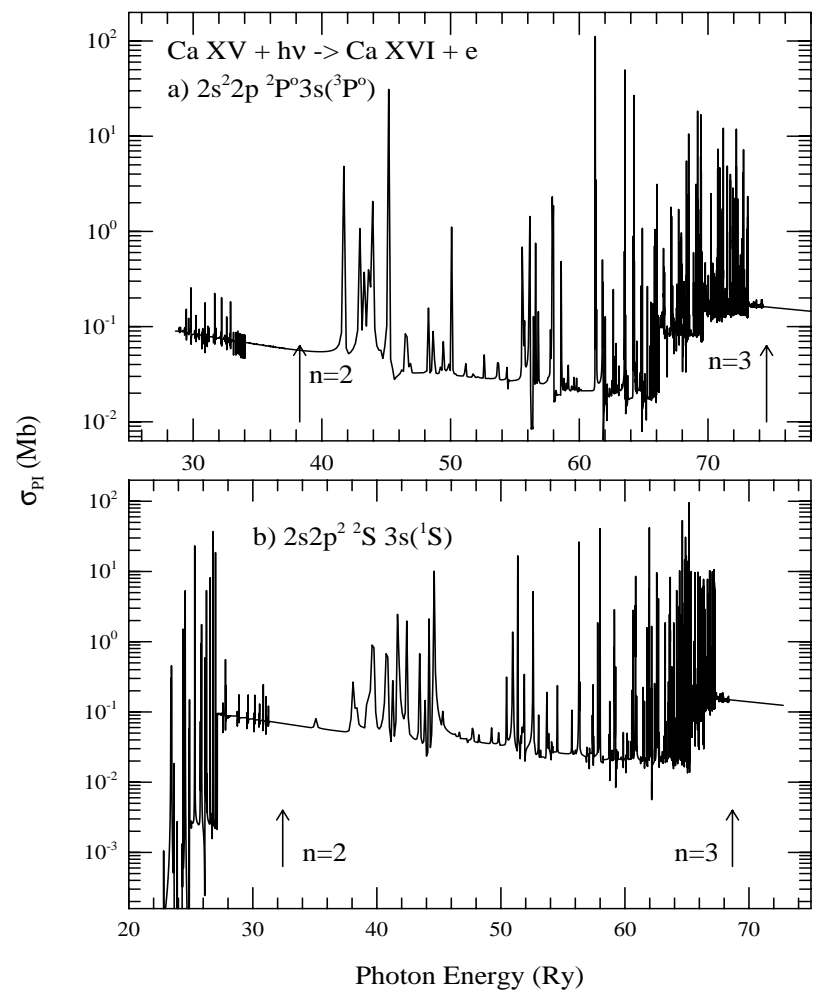

Figure 2: Photoionization cross sections $\sigma_{P I}$ of two low-lying excited states a) $2 s^{2} 2 p{ }^{2} P^{o} 3 s\left({ }^{3} P^{o}\right)$, b) $2 s 2 p^{2}{ }^{2} S 3 s\left({ }^{1} S\right)$ of Ca XV. They illustrate considerable impact, in terms of high peak resonances and enhanced background, by the core excitations to high lying autoionizing states of $n=3$ complex compared to those of $n=2$ complex. 


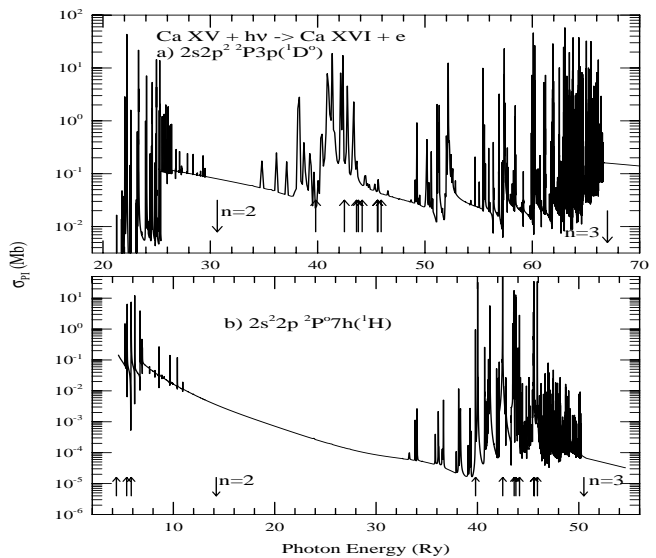

Figure 3: Photoionization cross sections $\sigma_{P I}$ of excited states, a) $2 s 2 p^{2}{ }^{2} P 3 p\left({ }^{1} D^{o}\right)$, b) $2 s^{2} 2 p^{2} P^{o} 7 h\left({ }^{1} H\right)$ of $\mathrm{Ca} \mathrm{XV}$ showing existence of strong Seaton resonances, positions pointed by arrows, due to core excitation to various dipole allowed states in the high energy region.

cited states with single valence electron is the relatively wide Seaton resonances. These resonances usually appear embedded in narrow Rydberg resonances and often as wider resonant structure with background enhancement. They become more distinct with higher excited states. They form by photo-excitation-of-core (PEC) $[22,11]$ from ground to dipole allowed states. The photon is absorbed by the core for the dipole allowed transition from the ground state while the outer electron remains as spectator. This is followed by the core dropping back to ground state while the outer electron is ejected out. Hence these resonances form at photon energy matching the excitation energy regardless of the ionization energy. Figure 3 presents $\sigma_{P I}$ of two excited levels, a) $\left.2 s 2 p^{2}{ }^{2} P\right) 3 p\left({ }^{1} D^{o}\right)$ and b) $2 s^{2} 2 p^{2} P^{o} 7 h\left({ }^{1} H\right)$ of $\mathrm{Ca} \mathrm{XV}$ where Seaton resonances are marked by arrows (positions determined by the energies in Table 1) pointing upward. It may be noted that the Seaton resonances for $\mathrm{n}=2$ complex exist for the state $\mathrm{b}$ ) $2 s^{2} 2 p^{2} P^{o} 7 h\left({ }^{1} H\right)$ but not for state $\left.2 s 2 p^{2}{ }^{2} P\right) 3 p\left({ }^{1} D^{o}\right.$ ) (a) because the later has ionization energy above the photon energy needed for PEC to $n=2$ states. The interference between Seaton resonances and Rydberg resonances can enhance the background considerably, as seen in Figure $3(\mathrm{a}, \mathrm{b})$. The ${ }^{1} D^{o}$ state (a) starts with almost zero cross sections and rises with energy, has the region between $\mathrm{n}=2$ and $\mathrm{n}=3$ states filled with high peak resonances and ends in enhanced background. For the highly excited state (b) $2 s^{2} 2 p^{2} P^{o} 7 h\left({ }^{1} H\right)$ the background decays until reaching PEC energies which start at 40 Ry when Seaton resonances form. It can be seen that the peaks higher than those for $n=2$ complex. These are the structures that can affect photoionization and recombination rates at high temperatures.

To assess the net contribution of resonant features and enhancements aris- 
ing due to excitations of the core to $\mathrm{n}=3$ states, integrated oscillator strength of photoionization cross sections of the two excited states, a) $2 s^{2} 2 p{ }^{2} P^{o} 3 s\left({ }^{3} P^{o}\right)$, b) $2 s 2 p^{2}{ }^{2} S 3 s\left({ }^{1} S\right)$, of Figure 2 have been computed with and without their contributions. The differential oscillator strength $\frac{d f}{d \epsilon}$ quantitatively relates to photoabsorption per unit energy in the bound-bound and the continuum region. In the bound-free region the integrated $d f / d \epsilon$ yields the effective photoabsorption in terms of $\sigma_{P I}$ including the autoionizing resonance (e.g. [23]), as

$$
\bar{f}_{r}=\int \frac{d f_{i}}{d \epsilon} d \epsilon=\frac{1}{4 \pi^{2} \alpha a_{0}^{2}} \int \sigma_{P I}(i, \epsilon) d \epsilon
$$

Considerable differences in terms of order of magnitude are found for the value of $\bar{f}_{r}$ with (29CC expansion) and without (8-CC expansion) inclusion high core excitations. The value of $\bar{f}_{r}$ is $7.611 \mathrm{e}-02$ with 8 -CC and 1.099 with $29-\mathrm{CC}$ for the ${ }^{1} S$ state, while it is $8.70 \mathrm{e}-02$ with 8 -CC and 1.814 with 29 -CC for the ${ }^{3} P^{o}$.

The present results should provide precise plasma modelings. As discussed above, present energies agree with the measured values within $1.5 \%$. Based on the energy comparison, large wavefunction expansion to include features in the high energy region, the typical accuracy of R-matrix method and close coupling the accuracy may range from 10 to $30 \%$ for various applications. While for high temperature plasmas the accuracy may be $10 \%$ for low temperature plasmas it could be $30 \%$ with the assumption that low energy resonances are important. LS coupling does not include resonances of intermediate couplings which may appear in fine structure. Inclusion of relativistic effects should produce more resolved resonances through couplings of fine structure levels as well as some additional resonances. However, the relativistic computations will be extensive since 29 LS states will split into over 60 fine structure levels, and thus increasing the size of the Hamiltonian matrix. The present results are expected to be accurate enough for practical purposes since the distribution functions in modelings is typically insensitive to high resolution of resonances in high energies. It may be mentioned that $29-\mathrm{CC}$ does not includes all possible states of $\mathrm{n}=3$ complex. Hence a larger than 29-CC expansion should introduce some additional resonances in the higher energy region. Those resonances may not be strong enough for any significant contribution since, as figures 2 and 3 show, there seems to be the trend of convergence of enhancement.

\section{Conclusion}

The work presents photoionization cross sections of 701 bound states of $\mathrm{Ca} \mathrm{XV}$ with the new features in the high energy region through consideration of a large wavefunction expansion of 29 core states. The study show extensive strong resonances and enhanced background cross sections due to core excitations to $n=3$ complex. In addition Seaton resonances show prominent existence in high energy region. The results should have significant contributions in photoionization and recombination rates at temperatures where Ca XV exist in stellar plasmas and hence in determination of abundance of $\mathrm{Ca}$ in these objects. 
These should also provide a more precise and complete modeling of astrophysical and laboratory applications. The present cross sections are expected to be of accuracy of about 10-30\% based on (i) good agreement of energies with the measured values, (ii) large number of core excitations, (iii) higher resolution for resonances, and (iv) consideration of large number configurations.

All photoionization data are available electronically from on-line NORADAtomic-Data page at (NaharOSURadiativeAtomicData) website:

http://norad.astronomy.ohio-state.edu/

Acknowledgments

This work was supported partially by NSF AST-1312441 and DOE DESC0012331. The computational work was carried out at the Ohio Supercomputer Center (OSC) in Columbus Ohio.

\section{References}

[1] Dere, K. P.: 1978, Astrophys. J. 221, 1062.

[2] Laming, J. M. and Drake, J. J.: 1999, Astrophys. J. 516, 324

[3] Keenan, F. P., Conlon, E. S., Foster, V. J., Aggarwal, K. M., and Widing, K. G.: 1992, Astrophys. J. 401 , 411

[4] Aggarwal, K. M. and Keenan, F. P.: 2003, Astron. Astrophys. 407, 769

[5] D Luo \& AK Pradhan, 1989, J. Phys. B, 22, 3377

[6] S.N. Nahar and A.K. Pradhan, Photoionization of highly charged carbonlike ions, Phys.Rev.A 45, 7887 (1992)

[7] M.J. Seaton, J. Phys. B 20, 6363-6378 (1987)

[8] The Opacity Project Team .The Opacity Project, Vol 1, 1995, Vol. 2, 1996, Institute of Physics Publishing

[9] S.N. Nahar, A.K. Pradhan, G.X. Chen, W. Eissner,. "Highly Excited Core Resonances in Photoionization of Fe xvii : Implications for Plasma Opacities", Phys. Rev. A 83, 053417 (2011)

[10] S.N. Nahar, A.K. Pradhan, Phys.Rev.Lett 116, 235003 (2016)

[11] A.K. Pradhan and S.N. Nahar, Atomic Astrophysics and Spectroscopy (Cambridge University press, 2011)

[12] P.G. Burke, W.D. Robb, Adv. At. Mol. Phys. 11, 143-214 (1975)

[13] D.G. Hummer, K.A. Berrington, W. Eissner, A.K. Pradhan, H. Saraph, J.A. Tully, Astron. Astrophys. 279:298-309 (1993) 
[14] K.A. Berrington, P.G. Burke, K. Butler, M.J. Seaton, P.J. Storey, K.T. Taylor, Y. Yu, J. Phys. B 20,6379-6397 (1987)

[15] Berrington, K.A., Eissner, W., Norrington, P.H. ..RMATRIX1: Belfast atomic R-matrix codes. 1995, Comput. Phys. Commun. 92, 290-420

[16] S.N. Nahar, W. Eissner, G.X. Chen, A.K. Pradhan, A\&A 408, 789 (2003)

[17] W. Eissner, M. Jones, H. Nussbaumer, Comput. Phys. Commun. 8, 270 (1974)

[18] S.N. Nahar, "Photoionization cross sections and oscillator strengths for Fe III", Phys. Rev. A 53, 1545 (1996)

[19] J. Sugar and C. Corliss, J. Phys. Chem. Ref. Data 14, Suppl. 2, 1664 (1985)

[20] http://physics.nist.gov/PhysRefData/ASD/levels_form.html

[21] S.N. Nahar, A.K. Pradhan, H.L. Zhang, Astrophys. J. Suppl 133, 255 (2001)

[22] Y. Yu, M.J. Seaton, J. Phys. B 20, 6409 (1987)

[23] S.N. Nahar, A.K. Pradhan, Phys.Rev.A Rapid Commun. 63, 060701 (2001) 